\title{
Optimal band structure for thermoelectrics with realistic scattering and bands
}

\author{
Junsoo Park $\mathbb{D}^{1 凶}$, Yi Xia $\mathbb{D}^{2}$, Vidvuds Ozolinšs $\mathbb{B D}^{3,4}$ and Anubhav Jain $\mathbb{D}^{1 凶}$
}

Understanding how to optimize electronic band structures for thermoelectrics is a topic of long-standing interest in the community. Prior models have been limited to simplified bands and/or scattering models. In this study, we apply more rigorous scattering treatments to more realistic model band structures-upward-parabolic bands that inflect to an inverted-parabolic behaviorincluding cases of multiple bands. In contrast to common descriptors (e.g., quality factor and complexity factor), the degree to which multiple pockets improve thermoelectric performance is bounded by interband scattering and the relative shapes of the bands. We establish that extremely anisotropic "flat-and-dispersive" bands, although best-performing in theory, may not represent a promising design strategy in practice. Critically, we determine optimum bandwidth, dependent on temperature and lattice thermal conductivity, from perfect transport cutoffs that can in theory significantly boost $z T$ beyond the values attainable through intrinsic band structures alone. Our analysis should be widely useful as the thermoelectric research community eyes $z T>3$.

npj Computational Materials (2021)7:43; https://doi.org/10.1038/s41524-021-00512-w

\section{INTRODUCTION}

Thermoelectricity enables clean electricity generation and fluidfree cooling. The ultimate goal of basic thermoelectric materials research is to design or discover materials with high figure of merit $z T$, commonly expressed as:

$z T=\frac{a^{2} \sigma}{K_{e}+\kappa_{\text {lat }}} T$.

Here, the thermoelectric power factor (PF) is the product of Ohmic charge conductivity $(\sigma)$ and the Seebeck coefficient $(a)$ squared. The total thermal conductivity $\kappa$ is the sum of electronic thermal conductivity $\left(\kappa_{e}\right)$ and lattice thermal conductivity $\left(\kappa_{\text {lat }}\right)$. A major challenge in achieving high $z T$ and $P F$ is that the electronic transport quantities are linked by a set of anti-complementary correlations $^{1-11}: \sigma$ and $\kappa_{e}$ are positively correlated whereas $\sigma$ and $a$ are negatively correlated. Only $\kappa_{\text {lat }}$, a lattice property, is relatively independent, though it too exhibits some positive correlation with $\sigma$ through structural symmetry. These interrelations make it difficult to determine the effect of various design strategies to optimize $z T$.

Equations based on the single parabolic band (SPB) model often underpin intuition about thermoelectric behavior. However, they tacitly assume that there is always enough (infinite) dispersion in all directions to cover the entire energy range relevant to thermoelectric phenomena. Instead, in most cases of practical interest, a band's dispersion changes in curvature (e.g., from positive to negative), crosses the Brillouin zone (BZ) boundary orthogonally, and tops out at some maximum energy. In addition to band shape considerations, thermoelectric properties can widely vary depending on what is assumed of the scattering behavior. Typical models and descriptors assume a behavior that is dominated by intraband/intravalley, elastic acoustic phonon scattering, and can be derailed when other scattering mechanisms and interband/intervalley transitions have large effects ${ }^{12-16}$. Several studies have analytically investigated thermoelectricity using model band structures and scattering ${ }^{15-19}$, but they had one or more of the following limitations: (1) the bands were purely parabolic or parabolic-like with infinite dispersion; (2) only a single isotropic band was considered; (3) models for scattering and/or transport were based on constant lifetimes, constant mean free paths, or at best scattering proportional to the density of states (DOS).

To more generally addresses the topic of optimal band structure, we create more realistic model solid-state band structures and more faithfully model carrier scattering due to multiple sources. Our band structures are properly confined to a finite BZ with smooth inversion of upward (downward) parabolicity to downward (upward) parabolicity for describing conduction (valence) states-a key for retaining generality, physicality, and approximate compatibility with established scattering formalism. We modify established formulae for various scattering mechanisms-deformation-potential scattering (DPS), polar-optical scattering (POS), and ionized-impurity scattering (IIS) -as to capture the effects of inverted parabolicity, anisotropy, and band multiplicity on carrier lifetimes. Refer to "Methods" for further details. We monitor how thermoelectric properties of one or more bands respond to variations in band shapes (see Fig. 1). Our study fine-tunes conclusions drawn from simpler models on design strategies such as anisotropy, band multiplicity, and resonance levels. Finally, we determine the optimum bandwidths as a function of temperature and $\kappa_{\text {lat }}$, which improves $z T$ beyond what is normally accessible.

We start by rewriting Eq. (1) to better reflect fundamental transport relations

$z T=\frac{\left(\zeta^{2} / \sigma\right)}{K_{e}+K_{\text {lat }}} T$.

In Eq. (2), the key role is played by $\zeta$, a quantity for which there appears to be no conventional name. We refer to it as the "thermoelectric conductivity"; in the Onsager-Callen formulation

\footnotetext{
${ }^{1}$ Energy Technologies Area, Lawrence Berkeley National Laboratory, Berkeley, CA, USA. ${ }^{2}$ Department of Materials Science and Engineering, Northwestern University, Evanston, IL, USA. ${ }^{3}$ Department of Applied Physics, Yale University, New Haven, CT, USA. ${ }^{4}$ Energy Sciences Institute, Yale University, West Haven, CT, USA. ${ }^{凶}$ email: qkwnstn@gmail.com; ajain@lbl.gov
} 
a

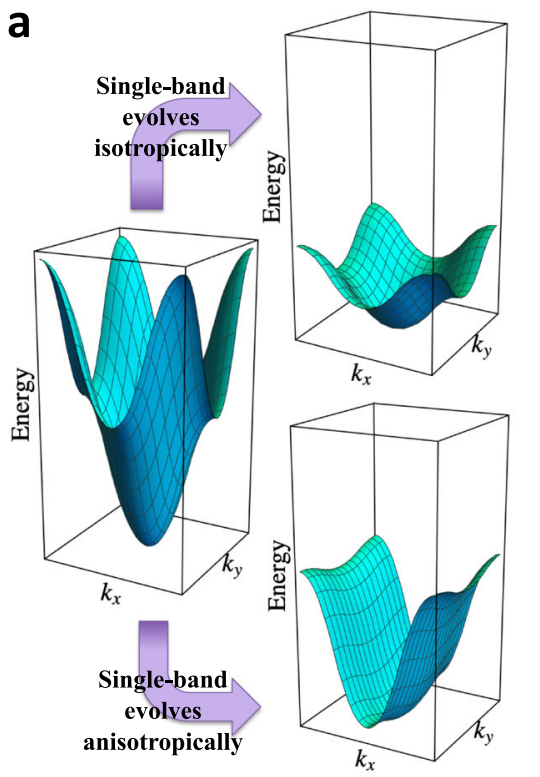

b

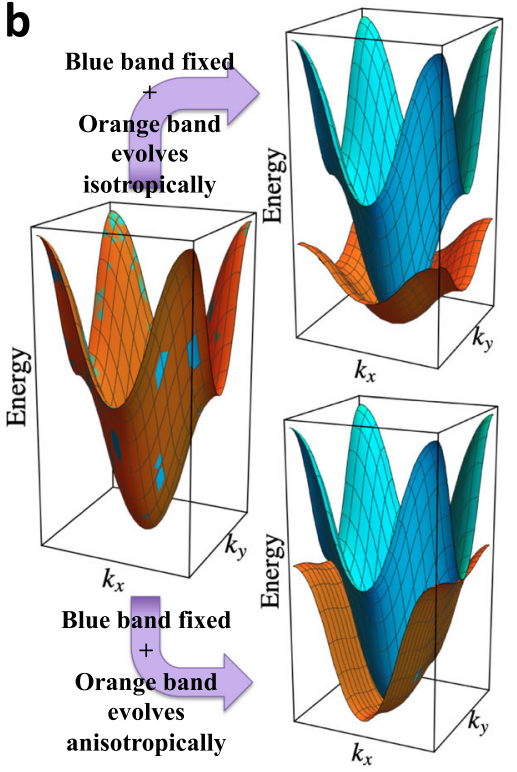

C

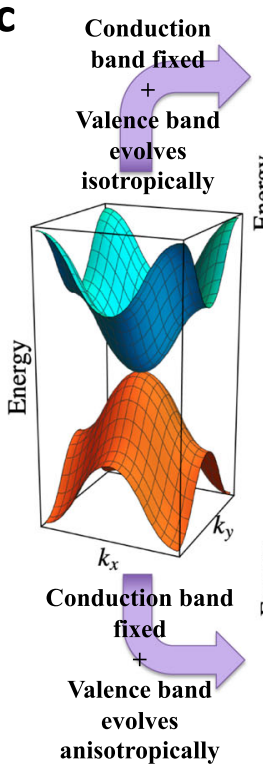

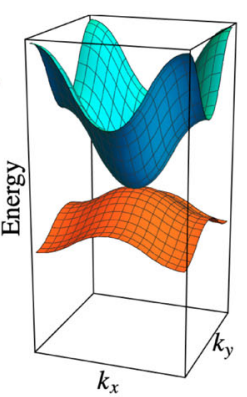

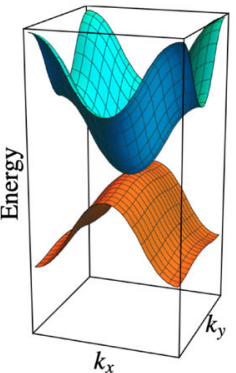

Fig. 1 The evolution of band structure models used in this study. a A single-band changes in effective mass. $\mathbf{b}$ One band (orange) changes in effective mass while another band (blue) is fixed. $\mathbf{c}$ The valence band (orange) changes in effective mass while the conduction band (blue) is fixed. Note that two-dimensional (2D) band structures are shown for graphical purposes. In the study where three-dimensional (3D) bands are used, two types of anisotropic evolution are considered: one where a band grows heavy in one direction and another where the band grows heavy in two directions. Each band is an upward paraboloid smoothly inflecting to an inverted paraboloid halfway to the BZ boundary.

of coupled charge-and-heat conduction ${ }^{20-22}, \zeta$ is the quantity responsible for the thermal-gradient-to-charge-current conversion $\left(\mathbf{J}_{c}=\sigma \mathbf{E}-\zeta \nabla T\right)$. That is, $\zeta$ represents the charge conductivity due to thermal driving force, the essence of thermoelectricity. Equation (2) lifts the hidden coupling between $a$ and $\sigma(a=\zeta / \sigma)$ and correctly identifies $\zeta$ as the quantity that must be high but that $\sigma$ must be low. That is, we desire high thermoelectric conductivity, not Ohmic conductivity - a correction to the routine but ambiguous thermoelectric adage that "electrical conductivity" must be high.

Insights into maximizing $z T$ are attained by examining Eq. (2) through Boltzmann transport formalism ${ }^{23-26}$,

$$
\sigma=\frac{1}{V} \int \Sigma(E)\left(-\frac{\partial f}{\partial E}\right) d E
$$

$\zeta=\frac{1}{V T} \int\left(E_{\mathrm{F}}-E\right) \Sigma(E)\left(-\frac{\partial f}{\partial E}\right) d E$

$\kappa_{e}=\frac{1}{V T} \int\left(E_{\mathrm{F}}-E\right)^{2} \Sigma(E)\left(-\frac{\partial f}{\partial E}\right) d E-\frac{\zeta^{2}}{\sigma} T$

where $V$ is the cell volume, $E_{\mathrm{F}}$ is the Fermi level, $f(E)$ is the Fermi-Dirac distribution, and $\Sigma(E)=v^{2}(E) \tau(E) D(E)$ is the spectral conductivity, composed of group velocity $(v)$, lifetime $(\tau)$, and DOS $(D)$. The three integrands share in common the term $\Sigma(E)\left(-\frac{\partial f}{\partial E}\right)$, the source of the positive correlations between $\sigma, \zeta$, and $k_{e}$. The integrands differ only in the power relation $\left(E_{\mathrm{F}}-E\right)^{p}$ as $p=0,1,2$. This juxtaposition states that, in relative terms, low-energy carriers contribute most to $\sigma$, high-energy carriers contribute most to $K_{e}$, while it is the medium-energy carriers that are most responsible for $\zeta$. That is to say, if one wished to increase $\zeta$ relative to $\sigma$ and $\kappa_{e}$, then $\Sigma(E)$ should be high in some medium-energy range and low elsewhere. The results that follow are interpreted with this picture in mind.

\section{RESULTS}

\section{Optimal performance-single band}

Here we investigate how a single band may yield the highest $z T$ with $E_{F}$ optimized for it. The performance is evaluated for different band structure shapes as depicted in the three "zones" of Fig. 2: (1) isotropic increase in $m,(2)$ anisotropic increase in $m$ in one direction ("unidirectional anisotropy"), and (3) anisotropic increase in $m$ in two directions ("bidirectional anisotropy"). The Seebeck coefficient predicted at a fixed $E_{F}$ is provided in Supplementary Discussion, which pinpoints how and why our model predictions deviate from the SPB model. The fluctuations in optimal $E_{F}$, displayed in Fig. 2a, is also analyzed there.

We first consider the case where $m$ varies isotropically (Zone 1 in Fig. 2b-d). As expected, a light band is definitely preferred: the PF and $z T$ both decrease with increase in $m$, as numerous studies agree upon ${ }^{27-29}$. A lighter band has higher mobility $(\mu)$ and thus is less needy of carrier concentration $(n)$ in providing a given value of $\sigma(\sigma=n \mu)$, which helps retain high $a$.

We observe that anisotropy is immensely beneficial (see Zones 2 and 3 in Fig. 2). Because DPS is almost exactly proportional to DOS, the performance under DPS is a clear indicator of the important role played by the energy-dependence of group velocity, $\left\langle v^{2}(E)\right\rangle$, which steepens with band anisotropy to enhance performance. See Supplementary Fig. 6 for the schematic. Steepening $\left\langle v^{2}(E)\right\rangle$ increases $\zeta$ over $\sigma$, simultaneously lowering optimal $E_{F}$. We make three major observations. First, in terms of $z T$, bidirectional anisotropy (one light, two heavy directions) outperforms unidirectional anisotropy (two light, one heavy direction). This is because $\left\langle v^{2}(E)\right\rangle$ in the former evolves to a one-dimensionallike profile, which is steeper than the two-dimensional-like profile that $\left\langle v^{2}(E)\right\rangle$ evolves to in the latter. Second, toward the extreme limit, both types of anisotropy plateau in performance. This occurs for two reasons: for one, $\left\langle v^{2}(E)\right\rangle$ converges to the respective lowdimensional linear limits, and for two, extreme anisotropy exhausts "low-energy voids". Refer to Supplementary Discussion for details. Third, because IIS and POS are less dependent on $D(E)$ than DPS, anisotropy is even more beneficial when they are the dominant mechanisms. Eventually though, because DPS increases 

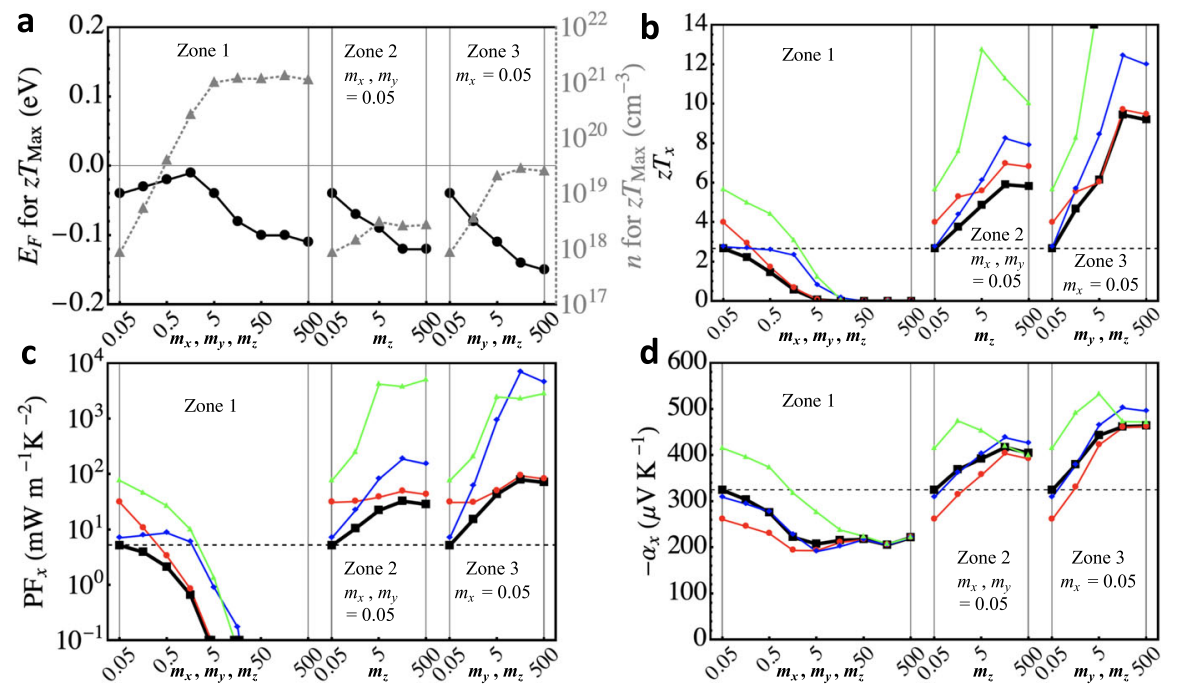

Fig. 2 Single-band thermoelectric properties in the light direction $(x)$ with $m_{x}=0.05$ with respect to its effective-mass profile. a Fermi level and carrier concentrations at optimum $z T$, $\mathbf{b}$ optimum $z T, \mathbf{c}$ the power factor, $\mathbf{d}$ and the Seebeck coefficient, in the $x$-direction. Each zone (as enclosed by vertical gray lines) indicates certain characteristic evolution: isotropic increase in $m$ from 0.05 to 500 in Zone 1 , anisotropic increase in $m_{y}$ from 0.05 to 500 in Zone 2, anisotropic increase in both $m_{y}$ and $m_{z}$ in Zone 3 from 0.05 to 500 . Four different scattering regimes are considered: the POS limit (blue), the IIS limit (green), the DPS limit (red), and the overall effect (black). Supplementary results for $\sigma, \mu, \zeta, \kappa_{e}, L$, $z_{e} T$ are in Supplementary Fig. 8.

most rapidly with DOS, it becomes dominant as anisotropy grows large. Overall, we observe that anisotropy improves $z T$ by as much as a factor of 3 above the isotropic value.

Values in Fig. 2 would be lower if $m_{x}$ were larger and $\kappa_{\text {lat }}$ were higher. In Supplementary Fig. 7, we show under $m_{x}=0.1$ and $\kappa_{\text {lat }}=1 \mathrm{~W} \mathrm{~m}^{-1} \mathrm{~K}^{-1}, z T$ is limited to 5 rather than 9 , in a closer neighborhood of the state-of-the-art, but draw the same relative benefit from anisotropy.

\section{Optimal performance-multiple bands}

Realistic band structures often feature multiple bands near the Fermi level. One of the best designs known for increasing $\sigma$ without paying a penalty on $a$ is multiplicity of band pockets aligned in energy ${ }^{30-34}$. Band multiplicity comes in various forms; however, we therefore examine the effects of (i) multiplicity of identical bands, (ii) coexistence of inequivalent bands (with varying the second band shape), and (iii) bipolar transport in the presence of valence and conduction bands (with varying valence band shapes). These band structures are illustrated in Fig. 1b, c. As justified in "Methods", our modeling of interband/intervalley scattering (henceforth inter-scattering) expands the phase space owing to the additional band and uses the factor $s_{\text {int }}=0.5$ making it half as strong as intraband/intravalley scattering (henceforth intra-scattering). For comparison, we also provide results obtained with $s_{\text {int }}=0$ (no inter-scattering) in Supplementary Fig. 11.

We start from two identical bands with aligned band minima, the first of which is isotropic and fixed while the second band then evolves according to Fig. $1 \mathrm{~b} . E_{F}$ is again optimized for maximum $z T$. The results are plotted in Fig. 3. The left edge of Zone 1 for each plot, where the two bands are identical, represents symmetrydegenerate band pockets. This offers higher $z T$ and PF as compared to the case of a single band (Fig. 2) though less than by twofold. Two identical bands result in essentially identical $a$ whereas $\sigma$ draws benefits from doubled $n$ somewhat negated by inter-scattering. One question of interest is the effect of increasing the number of identical carrier pockets. It is generally known that the more pockets the better, though it is straightforward even from our simplified analysis that doubling their number does not double the PF or $z T$ due to inter-scattering. For $N_{v}$ band pockets, $n \propto N_{v}$ while $\tau \propto\left(1+s_{\text {int }}\left(N_{v}-1\right)\right)^{-1}$. Then $\sigma \propto N_{v}\left(1+s_{\text {int }}\left(N_{v}-1\right)\right)^{-1}$, which as $N_{v}$ grows saturates to $s_{\text {int }}^{-1}$. For example, with $s_{\text {int }}=0.5$ that we assume, the maximum PF gain even with an infinite number of identical pockets is a factor of 2 . In fact, if inter-scattering is somehow stronger than intra-scattering, $s_{\text {int }}>1$, then $N_{v}$ is detrimental. As such, the benefit of $N_{v}$ is bounded by the degree of inter-scattering, whose minimization should be a priority of multiband strategies. Furthermore, if $\kappa_{e}>>K_{\text {lat, }}$ then $N_{v}$ is rather unimportant for $z T$ because $\kappa_{e}$ increases as much as the PF. If $\kappa_{\text {lat }}=$ 0 , hypothetically, then $N_{v}$ would have no effect as it cancels exactly for the PF and $\kappa_{e}$.

Next, keeping the principal first band fixed in shape and maintaining $s_{\text {int }}=0.5$, we make the second band heavier. As it turns heavier isotropically (Zone 1 in Fig. 3), $z T$ and the PF increasingly suffer until they sink well below even the values that the fixed principal band alone generates (compared to Fig. 2). This means that non-symmetry-related, accidentally degenerate pockets harm $z T$ if their band masses in the transport direction $\left(m_{x}\right)$ are sufficiently different. Two main reasons account for this. As the second band grows heavier in the transport direction $(x)$, its direct contribution to transport diminishes. It also indirectly sabotages the lighter principal band by triggering heavier inter-scattering overall. This holds until the second band becomes narrow enough for it to function as a resonance level and selectively scatter lowenergy carriers, which reduces $\sigma$ relative to $\zeta$, whereby $z T$ and the $P F$ rebound. They do not fully recover the values generated by the original twin degenerate bands unless DPS or POS dominates. The presence of strong IIS, due to the high impurity concentration required for doping a very heavy band, could eclipse the resonance level effect from manifesting.

If the second band evolves anisotropically in the $y$ and/or the $z$ directions, the thermoelectric response is largely similar to what is seen for a single-band turning anisotropic. Anisotropy increases $a$ and the PF as well as $z T$ until they plateau. Also, $z T$ is not noticeably higher here than in the case of a single anisotropic band because the anisotropic band dominates transport and $\kappa_{e}>>K_{\text {lat }}$. This again is a nod to the decreasing importance of the band multiplicity if $\kappa_{e}>>K_{\text {lat }}$.

Another two-band situation is a semimetallic one in which there exists a "conduction band" and a "valence band" with no gap in between, triggering bipolar transport. Bipolar effect is a significant suppressor of the Seebeck coefficients of metals and small-gap 

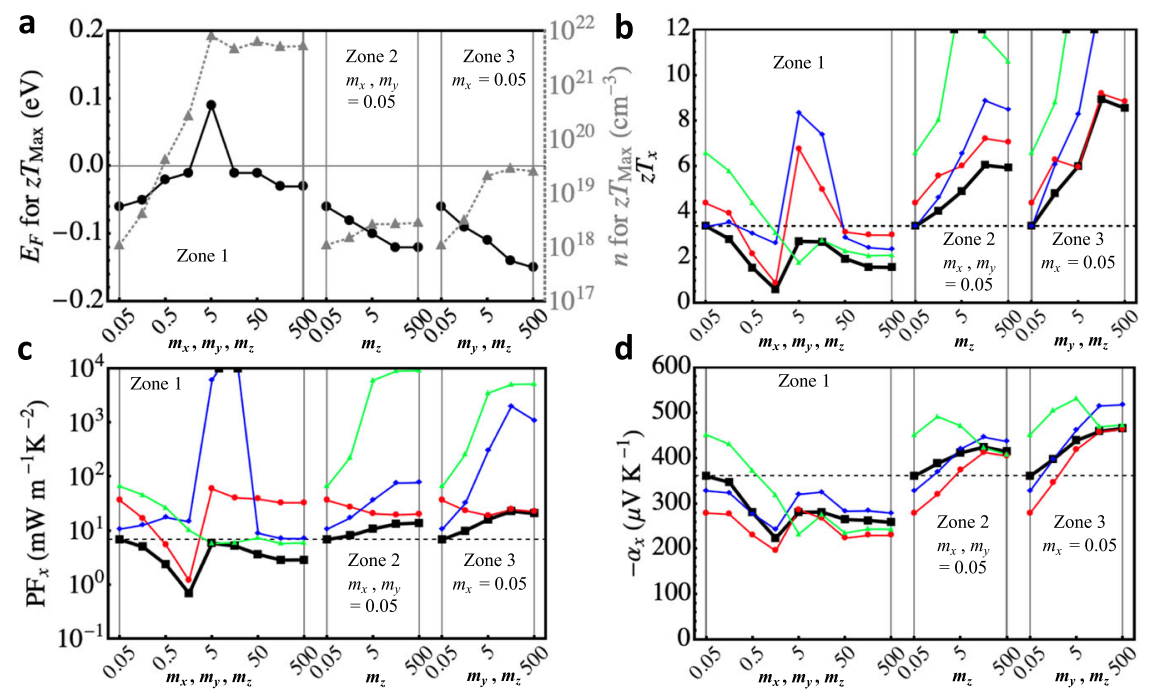

Fig. 3 Two-band thermoelectric properties in the light direction $(x)$ with respect to the evolution the second band's effective-mass profile while the first band is fixed at $\boldsymbol{m}_{\boldsymbol{x}}=\boldsymbol{m}_{\boldsymbol{y}}=\boldsymbol{m}_{\mathbf{z}}=\mathbf{0 . 0 5}$ with $\boldsymbol{s}_{\text {int }}=\mathbf{0 . 5}$. a Fermi level and carrier concentrations for optimum $z T$, b optimum $z T$, c the power factor, $\mathbf{d}$ and the Seebeck coefficient, in the $x$-direction. Each zone (as enclosed by vertical gray lines) indicates certain characteristic evolution of the second band: isotropic increase in $m$ from 0.05 to 500 in Zone 1, anisotropic increase in $m_{y}$ from 0.05 to 500 in Zone 2, anisotropic increase in both $m_{y}$ and $m_{z}$ in Zone 3 from 0.05 to 500 . Four different scattering regimes are considered: the POS limit (blue), the IIS limit (green), the DPS limit (red), and the overall effect (black). Supplementary results $\sigma, \mu, \zeta, K_{e}, L, z_{e} T$ are in Supplementary Fig. 10.
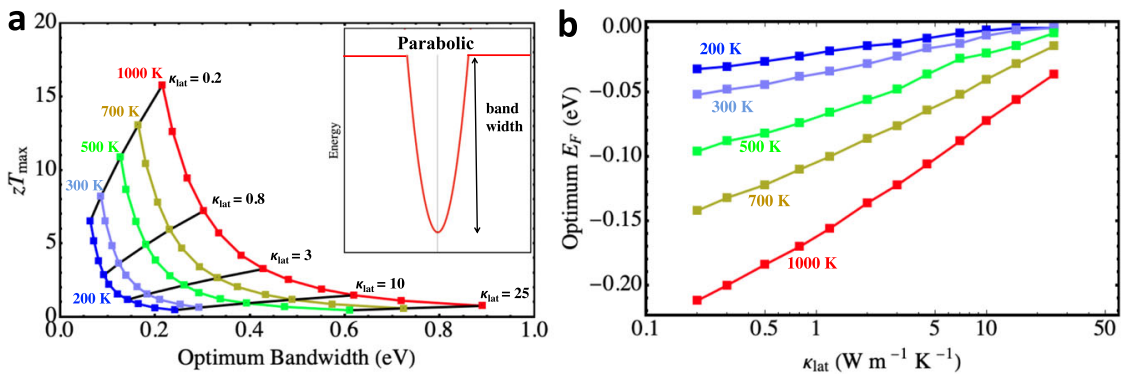

Fig. 4 Optimum bandwidth, Fermi level, and $z T$. a $T$ - and $\kappa_{\text {lat }}$-dependent optimum bandwidth and $z T$ under DPS for an isotropic 3D parabolic band of $m_{\mathrm{GaAs}}=0.067$, and $\mathbf{b}$ the optimum Fermi level for each point. The lower the $\kappa_{\mathrm{lat}}$, the lower the optimum $E_{F}$ and $W_{\mathrm{opt}} \kappa_{\mathrm{lat}}$ is given in $\mathrm{W} \mathrm{m} \mathrm{m}^{-1} \mathrm{~K}^{-1}$.

semiconductors. Extrapolating the lessons from above, it is rather straightforward that for $\zeta$ to be large in magnitude (positive or negative), $\Sigma(E)$ must be highly asymmetric about the Fermi level, juxtaposing mobile and anisotropic "conduction" bands against isotropically heavy "valence" bands or vice-versa. We confirm this by fixing the conduction band and evolving the valence band as described in Fig. 1c. The results are in Supplementary Fig. 12 and "Discussion". It is therefore no surprise that high-performing semimetals and narrow-gap semiconductors feature quite drastic band asymmetries about the Fermi level ${ }^{35-40}$.

\section{Optimum bandwidth from perfect transport cutoff}

The relative energy ranges from which $\sigma, \zeta$, and $\kappa_{e}$ draw contributions imply that the best performance would be obtained by suppressing both low-energy contributions (to suppress Ohmic current) and high-energy contributions (to suppress thermal current) thus limiting transport only to a certain medium-energy range. Accordingly, we investigate the scenario in which the contribution to transport abruptly vanishes at some optimum energy (see the inset in Fig. 4a), which we define as the optimum bandwidth $\left(W_{\text {opt }}\right)$. It essentially represents optimum transport distribution width. Mathematically, $W_{\text {opt }}$ is obtained by solving for the following as to maximize $z T$ :

$$
W_{\text {opt }}=\underset{W}{\arg \max }\left[\frac{\zeta^{2}(W) / \sigma(W)}{\kappa_{e}(W)+\kappa_{\text {lat }}} T\right]
$$

where, for instance, $\sigma(W)=\frac{1}{V} \int_{0}^{W} \Sigma(E)\left(-\frac{\partial f}{\partial E}\right) d E$. Finite bandwidth of our definition would arise for a band that is abruptly crossed by numerous perfect energy-filtering states acting as perfect resonance levels, or a band that sharply and discontinuously flattens out. Admittedly neither is achievable to perfection in real life, but that theoretical limit is of our interest. We consider an isotropic parabolic band under DPS and optimize $E_{F}$. Unlike previous studies, we find that there does exist finite, optimum bandwidth for thermoelectrics that depends on temperature and $\kappa_{\text {lat }}$, as delineated by Fig. 4 .

Achieving $W_{\text {opt }}$ would be a tremendous boost for $z T$. Assuming $\kappa_{\text {lat }}<0.5 \mathrm{~W} \mathrm{~m}^{-1} \mathrm{~K}^{-1}$ and $m=0.067, W_{\text {opt }}$ elevates $z T$ well beyond 10 - higher than any value attainable through any plain band structures of the previous sections. For given $\kappa_{\text {lat }}, W_{\text {opt }}$ generally increases with temperature, as expected from the larger range of carrier excitation at higher temperatures. This implies that achieving $W_{\text {opt }}$ is particularly consequential for low temperatures $(T \leq 300 \mathrm{~K})$ where a difference of $0.1-0.2 \mathrm{eV}$ can force a shift in $z T$ by nearly an order of magnitude. As $\kappa_{\text {lat }}$ vanishes, $W_{\text {opt }}$ also vanishes, and $z T$ 
diverges. This would be the Mahan-Sofo limit, named after their seminal work that deduced widthless band to be optimal, if $\kappa_{\text {lat }}=$ $0^{41}$. Our recovery of this limit is also evidenced by Supplementary Fig. 8f, in which the "electronic-part" $z T$, labeled $z_{e} T$, diverges to infinity as the band completely flattens out. In the other extreme, as $\kappa_{\text {lat }}$ becomes very high, $W_{\text {opt }}$ diverges, i.s., it is virtually irrelevant for $z T$. Further analysis is provided in Supplementary Discussion.

\section{DISCUSSION}

Commonly used descriptors for thermoelectric performance include the quality factor (QF), which under DPS is ${ }^{42,43}$

$\beta=T \frac{2 k_{\mathrm{B}}^{2}}{3 \pi} \frac{\rho v_{s}^{2} N_{v}}{m \Delta^{2} \kappa_{\text {lat }}}$,

and the Fermi surface complexity factor ${ }^{29}$

$C=N_{v}\left(\frac{2}{3}\left(\frac{m_{\perp}}{m_{\|}}\right)^{-\frac{1}{3}}+\frac{1}{3}\left(\frac{m_{\perp}}{m_{\|}}\right)^{\frac{2}{3}}\right)^{3 / 2}$.

Both metrics promote small effective mass ( $m$ or $m_{\|}$) and high band multiplicity $\left(N_{v}\right)$; the latter further promotes band anisotropy $\left(\frac{m_{\perp}}{m_{\|}}\right)$. This study serves as a general assessment of these wellknown blueprints in thermoelectrics, confirming some while offering fresh perspectives and more complete physical pictures to others.

(1) Small $m$ in the transport direction is always better.

(2) Band anisotropy is very beneficial, but the extent depends on its type. The advantage of anisotropy draws largely from the fact that $\left\langle v^{2}(E)\right\rangle$ rises to steeper, low-dimensional slopes. Bidirectional anisotropy mimicking 1D band structure is particularly beneficial, capable of increasing maximum $z T$ by nearly threefold for a given $m$ in the light direction.

(3) Although not captured by Eqs. (7) or (8), the gains from pocket multiplicity and band convergence depend on the relative shapes of the bands and what is assumed of interband scattering. A heavier pocket in the presence of a lighter pocket can be detrimental. Metrics such as QF or $C$ always predict better performance in the presence of more bands because they do not possess any component that accounts for inter-scattering or differential intrinsic transport of each bands. The metrics ought take these effects into account by bounding the gain from $N_{v}$ e.g., using a term such as $N_{v}\left(1+s_{\text {int }}\left(N_{v}-1\right)\right)^{-1}$ as was previously described.

(4) Within the limits of our investigation, the type of scattering mechanism does not play a pivotal role in determining what band structure is optimal for $z T$, except in the context of resonance levels. In other words, the best-performing band structure is for the most part the same under DPS, POS, or IIS. The type of scattering decides how much $z T$ improves or suffers as a band transforms, but no transformation is decisively beneficial under one scattering regime but decisively detrimental under another. As one exception, resonance levels are beneficial if the dominant scattering mechanism is efficient at energy-filtering-DPS or POS. If an ineffective filtering mechanism, such as IIS, activates comparably or dominates, then resonance levels lose merit.

(5) There exist optimum bandwidths to a plain parabolic band if the transport contribution can be, albeit hypothetically, abruptly curtailed at some energy. Optimum bandwidth arises because low-energy states are undesired owing to their large contribution to $\sigma$ and high-energy states are undesired owing to their large contribution to $k_{e}$. W opt for a given $m$ depends on temperature and $\kappa_{\text {lat }}$. It is small $(<0.3 \mathrm{eV})$ so long as $\kappa_{\text {lat }}<1 \mathrm{~W} \mathrm{~m}^{-1} \mathrm{~K}^{-1}$, and can push $z T$ beyond what is normally accessible.
We stress that our investigation of optimum bandwidth has distinct characteristics from previous investigations in terms of both approach and conclusion. Mahan and Sofo deduced that a fully localized, widthless transport distribution (a completely flat band) would deliver maximal thermoelectric performance ${ }^{41}$, but under the assumption that $\kappa_{\text {lat }}=0$. Because $\kappa_{\text {lat }}>0$ in real materials, a widthless band and transport distribution would yield $z T=0$ as $v(E)$ and the PF vanish alike (see Fig. 2b Zone 1). In later studies of optimum bandwidth, the "full-width" definition of bandwidth, $E_{\max }-E_{\min }$, was adopted ${ }^{18,19}$. A major limitation of this set-up is that the full-width is inherently coupled with $m$ or the size of the BZ. Because smaller $m$ in the transport direction is always beneficial, bandwidth optimality must be probed independently under a fixed $m$, as done in this study. Indeed, in ref. ${ }^{18}$, it was determined that optimum fullwidth does not exist (is infinite) under $\tau(E) \propto D^{-1}(E)$ as $z T$ continues to increase with larger full-width, likely due to concomitantly decreasing $\mathrm{m}$. In contrast to these studies, we herein find temperature-dependent, finite optimum bandwidth in the presence of finite $\kappa_{\text {lat }}$. Our bandwidth represents scenarios whereby a band flattens abruptly or features such as high-energy resonance levels are engineered. Our conclusions are more practically relevant than vanishing bandwidth under zero $\kappa_{\text {lat }}$ or infinite full-width that is coupled with $m$ or the BZ size.

(6) Though more of a philosophical point, we propose that analysis of thermoelectrics be more frequently framed in terms of the "thermoelectric conductivity", $\zeta$, which offers more straightforward insights than that framed in terms of the Seebeck coefficient and Ohmic conductivity. By juxtaposing $\zeta$ against $\sigma$ and $\kappa_{e}$, it becomes clear that a band must develop high $\Sigma(E)$ in the mid-energy region to be optimal for thermoelectric application. Our finding of finite optimum bandwidth resonates with this intuition.

Reflection on real materials is also in order. In spite of the theoretically remarkable performance of extremely anisotropic, "flat-and-dispersive band structures", they in practice would be subject to a disadvantage due to polycrystallinity of commercialscale materials as well as symmetry considerations. Indeed, no candidate materials thus far not achieved the high $z T$ modeled here, and we project why in light of our modeling. We distinguish bands in a cubic cell from those in a non-cubic cell.

In a non-cubic cell, a flat-and-dispersive band limits light transport to only certain direction(s). Assuming polycrystallinity, conductivity through a series of differently oriented grains is best described by the lower Wiener bound for composite media, i.e., the harmonic average of directional conductivities ${ }^{44}$. Due to poor conductivities along the heavy branch(es), the harmonic average seriously hampers the overall performance under our model, as described in Supplementary Fig. 9. An anisotropic band is then never as good as its isotropic counterpart whose polycrystallineaveraged conductivities are identical to those in any principal direction.

$\mathrm{Bi}_{2} \mathrm{PdO}_{4}{ }^{45}$ and $\mathrm{BaPdS}_{2}{ }^{46}$ are good examples as neither compound is cubic but exhibit bidirectional-anisotropic flat-anddispersive valence bands. The DOS profiles are characterized by peak-like protrusions near the band edges followed by decays, confirming the 1D-like band structure. Polycrystalline $\mathrm{Bi}_{2} \mathrm{PdO}_{4}$ has been experimentally synthesized and investigated, but recorded rather disappointing $p$-type PF $\left(1 \mathrm{~mW} \mathrm{~m}^{-1} \mathrm{~K}^{-2}\right)$ and $z T(0.06)^{47}$. Because electronic transport is mobile only in one direction and inhibited in the two heavy directions by design, it is unlikely that their presumably high thermoelectric potential in the light direction would shine through unless the sample is a single crystal. $\mathrm{BaPdS}_{2}$ has not yet been tested, but it is reasonable to hypothesize that it may exhibit a similar behavior. 
Under cubic symmetry, all three principal directions are guaranteed the same number of light and heavy branches. Polycrystallinity may then be irrelevant here, but now the concern is that the coexistence of light and heavy branches in the direction of transport (as opposed to light in the transport direction, heavy in other directions) with inter-scattering between them can be inherently limiting (recall Zone 1 in Fig. 3). Relevant cases are Febased full-Heuslers ${ }^{48}$ and perovskite $\mathrm{SrTiO}_{3}{ }^{49}$, which exhibit unidirectional flat-and-dispersive conduction bands, with the 2Dlike, precipitous DOS at the band edge.

$\mathrm{Fe}_{2} \mathrm{TiSi}$, a member of the former family, is particularly intriguing because its flat-and-dispersive conduction bands are opposed by triply degenerate, isotropic valence bands, offering a direct comparison of the $n$-type and $p$-type performances of the respective band structures. According to our $\mathrm{DFT}^{50}$ calculation using the PBE functional ${ }^{51}$, the lowermost conduction band is very flat along $\Gamma-X$ whose energy width is $0.05 \mathrm{eV}\left(m_{\|} \approx 41\right)$ and dispersive in other directions $\left(m_{\perp} \approx 0.7\right)$. A second isotropic conduction band $(m \approx 0.4)$ is degenerate at $\Gamma$. Opposing them are three isotropic valence bands with comparable $0.4 \leq m \leq 0.75$. Theoretical thermoelectric properties were studied with rigorous first-principles treatment of electron-phonon scattering ${ }^{52}$, but the $n$-type PF $\left(5 \mathrm{~mW} \mathrm{~m}^{-1} \mathrm{~K}^{-2}\right.$ at $300 \mathrm{~K}$ ) was predicted to be only barely higher than the $p$-type PF $\left(4 \mathrm{~mW} \mathrm{~m}^{-1} \mathrm{~K}^{-2}\right.$ at $\left.300 \mathrm{~K}\right)$, with no sign of the pronounced performance promised by the flat-and-dispersiveness.

As for $\mathrm{SrTiO}_{3}$, according to our DFT calculation, the width of the heavy branch of the lowermost conduction band along $\Gamma-X$ is $\sim 0.1 \mathrm{eV}\left(m_{\|} \approx 7\right)$ while in the dispersive directions $m_{\perp} \approx 0.8$. Two additional relatively isotropic conduction bands $(0.4 \leq m \leq 0.7)$ disperse from $\Gamma$ at the CBM. Multiple experimental reports exist for $\mathrm{SrTiO}_{3}$ on single crystals, which should be the best-performing and the most comparable to theory results. Although respectable $n$ type PFs of $3.6^{53}$ and $2.3 \mathrm{~mW} \mathrm{~m}^{-1} \mathrm{~K}^{-254}$ have been recorded at room temperature, neither are these values anywhere near what Fig. 2 promises.

These observations collectively suggest that cubic symmetry may cap the full potential of the flat-and-dispersive bands in real materials. As a separate point, it would certainly help if the band masses in the light direction of the both compounds were much smaller.

As a final deliberation, we address the question, what then is the optimal band structure, all things considered? The literature has convincing cases for both an extremely anisotropic, flat-anddispersive band and a band with multiple dispersive pockets at off-symmetry points. Reflecting on our modeling, we conclude the following. For a single band, if a bidirectional flat-and-dispersive band attaining $\frac{m_{\perp}}{m_{\|}} \approx 1000$ with as small a possible $m_{\|}$can be realized in a single crystal, it would constitute the optimal singleband structure. Otherwise, a band with multitude of dispersive pockets at off-symmetry points with weak inter-scattering would be the best targets, as they provide moderate anisotropy and would be more immune to polycrystallinity. For a given single band, presence of additional bands with equally light mass in the transport direction would increases performance, though this benefit is negligible if $\kappa_{e}>K_{\text {lat }}$ and/or if $s_{\text {int }} \approx 1$. For a given overall intrinsic band structure, resonance levels and optimum bandwidth further improve performance, the latter being capable of boosting $z T$ to the highest values of all band structure designs considered here and particularly consequential for low-temperature operation.

As efforts to discover and design thermoelectrics with $z T>3$ continue, the blueprints for high performance grow increasingly influential. Common rules regarding beneficial band structures for bulk thermoelectrics are largely drawn from simple band models without realistic scattering. Using a straightforward but improved approach, we herein fine-tune those blueprints while proposing optimal band structures and design principles along the way. Our generalized findings from this modeling study are mutually supportive of and consistent with the findings from recent targeted studies of high-performing materials with high-fidelity first-principles computations ${ }^{35,37,52,55,56}$. We hope that the theoretical investigations of the present study help the community navigate rationally toward next-generation thermoelectrics.

\section{METHODS}

The Hartree atomic units $\left(\hbar=m_{e}=a_{o}=q=4 \pi \epsilon_{0}=1\right)$ are used throughout "Methods." All calculations are performed on a set of in-house Mathematica codes.

\section{Band structure}

To generate a realistic solid-state band structure, a band is created by smoothly connecting an upward paraboloid to an inverted paraboloid at a selected inflection point. The advantages of such a band structure include: (1) it remains faithful to solid-state band theory that requires a band to cross the zone-boundary orthogonally, save for when crystal and orbital symmetries allow band crossing or degeneracy at the zone boundary (e.g., in graphene); (2) it formally maintains the validity of effective-mass-based description of the band throughout; (3) relatively simple analytic models of scattering can be directly applied to the upward-parabolic portion, and can be applied with modification for the inverted-parabolic portion; and (4) it can be used to explore a wide range of band structure shapes by modulating the points of inflection. The equation for this band structure is

$$
\begin{aligned}
E=E_{0} & +\left(\frac{\operatorname{Min}\left(\left|k_{x}\right|, \tilde{k}_{x}\right)}{2 m_{\text {up }, x}}+\frac{{\tilde{k_{x}}}^{2}-\left(G_{x}-\operatorname{Max}\left(\left|k_{x}\right|, \tilde{k}_{x}\right)\right)^{2}}{2 m_{\text {down }, x}}\right) \\
& +\left(\frac{\operatorname{Min}\left(\left|k_{y}\right|, \tilde{k}_{y}\right)}{2 m_{\text {up }, y}}+\frac{{\tilde{k_{y}}}^{2}-\left(G_{y}-\operatorname{Max}\left(\left|k_{y}\right|, \tilde{k_{y}}\right)\right)^{2}}{2 m_{\text {down }, y}}\right) \\
& +\left(\frac{\operatorname{Min}\left(\left|k_{z}\right|, \tilde{k}_{z}\right)}{2 m_{\text {up }, z}}+\frac{{\tilde{k_{z}}}^{2}-\left(G_{z}-\operatorname{Max}\left(\left|k_{z}\right|, \tilde{k}_{z}\right)\right)^{2}}{2 m_{\text {down }, z}}\right) \\
& \pm\left(\frac{\left|\tilde{k}_{x}^{2}-\left(G_{x}-\tilde{k}_{x}^{2}\right)^{2}\right|}{2 m_{\text {down }, x}}+\frac{\left|\tilde{k}_{y}^{2}-\left(G_{y}-\tilde{k}_{y}^{2}\right)^{2}\right|}{2 m_{\text {down }, y}}+\frac{\left|\tilde{k}_{z}^{2}-\left(G_{z}-\tilde{k}_{z}^{2}\right)^{2}\right|}{2 m_{\text {down }, z}}\right) .
\end{aligned}
$$

Here, $\tilde{k}_{x}$ denotes the inflection point in the $x$-direction, and $G_{x}$ is the reciprocal lattice vector in the $x$-direction, i.e., the $B Z$ boundary in the $x$-direction. If inflection occurs halfway to the $B Z$ boundary, then $\tilde{k}_{x}=G_{x} / 2$. The last terms are subtracted $(-)$ if $\tilde{k}_{x} \geq G_{x} / 2$, and added $(+)$ if $\tilde{k}_{x}<G_{x} / 2$. The same is true in the $y$ and the $z$ directions. Effective masses of the inverted-parabolic portion $\left(m_{\text {down }}\right)$ are obtained by enforcing derivative continuity at the inflection point in every direction.

Although a broad range of band shapes could be explored by changing the inflection point $\tilde{k}$ in any three Cartesian directions, in this work we limit ourselves to bands that inflect halfway to the zone boundary in all three directions. Under this assumption, the effective mass (inverse curvature) profiles of the upward paraboloid portion and the inverted paraboloid portion are identical $\left(m_{\text {up }}=m_{\text {down }}\right)$, rendering the entire band structure describable with one common set of directional effective masses. We note that this band could also serve as a first-order approximation of the tight-binding cosine band. We create these model bands centered at $\Gamma$ in a simple cubic Brillouin zone corresponding to an arbitrary lattice parameter of $15 a_{o}$ (Bohr radius) $7.9 \AA$, which is a reasonable lattice parameter for a real thermoelectric. The DOS is calculated using the tetrahedron method ${ }^{57}$. Detailed diagrams of the band structure and DOS are given in Supplementary Figs. 1 and 2.

\section{Carrier scattering and transport}

Thermoelectric properties are computed by numerically integrating Eqs. (3)-(5). The BZ is sampled to convergence with a k-point mesh of $40 \times$ $40 \times 40$. We fix the effective mass of the principal band in the transport direction $\left(m_{x}\right)$, unless it evolves isotropically, to enable fair comparison of performance of various band structures. We also ignore bipolar transport except in the two-band case with a conduction and a valence band. This is roughly tantamount to assuming a band gap larger than $0.4 \mathrm{eV}$-the maximum energy range of thermal excitation when the Fermi level is placed at the band minimum.

The ultimate objective is to determine band structures that theoretically maximize thermoelectric performance, and for that, some settings in place are those known to be beneficial for thermoelectrics. For instance, we intentionally fix $\kappa_{\text {lat }}$ to a low value of $0.5 \mathrm{~W} \mathrm{~m}^{-1} \mathrm{~K}^{-1}$ as is the case in many phonon-glass materials ${ }^{58-62}$. We fix the principal band mass in the transport direction $\left(m_{x}\right)$ to a small value of 0.05 , in the range of GaAs $(0.067)$ and $\mathrm{InSb}(0.014)$. Band anisotropy is also taken to the extreme to 
explore the limits of its benefits. These sometimes lead to prediction of higher $z T(\sim 9)$ than commonly encountered in the literature. Though these settings are optimistic and difficult to simultaneously satisfy experimentally, they are far from unrealistic, as materials with lower $m$ or $k_{\text {lat }}$ are known. They constitute the right regime of exploration in the discourse of high-performance thermoelectrics, and provide some estimate of the realistic upper limit of bulk thermoelectric performance.

To calculate $\tau$, various scattering mechanisms, namely DPS by (acoustic) phonons, POS, and IIS, are treated according to well-established formalisms ${ }^{24,63-67}$ with appropriate adjustments to account for anisotropy, inverted parabolicity and the BZ-bounded nature of our band structures, with details to follow in the next subsections. Once the lifetimes under the three mechanisms are calculated, the overall $\tau$ is estimated by Matthiesen's rule ${ }^{68}$.

Supplementary Fig. 3 plots the scattering profiles of the three processes based on Eqs. (10), (12), and (13). For the isotropic cases, they exhibit excellent agreement with the corresponding parabolic band formulations. DPS exhibits the expected $\tau^{-1} \propto D(E)$ relation. POS is characterized by the emission onset near the band edge followed by a gradual decay. IIS reproduces the Brooks-Herring behavior.

\section{Deformation-potential scattering (DPS)}

The theory of deformation potential was originally developed by Bardeen and Shockley for long-wavelength acoustic phonon scattering with the assumption of elasticity ${ }^{69}$. Combining it with the generalized deformation potential by Kahn and Allen ${ }^{70}$, we write the DPS lifetimes as follows:

$$
\tau_{\mathrm{DPS}, \mathbf{k}}=\frac{\rho v_{s}^{2}}{\pi k_{\mathrm{B}} T\left(\Delta+m \mathbf{v}_{\mathbf{k}} \cdot \mathbf{v}_{\mathbf{k}}\right)^{2}} D^{-1}\left(E_{\mathbf{k}}\right),
$$

where $\Delta$ is the Bardeen-Shockley deformation potential at the band edge (band shift with lattice deformation) and the Kahn-Allen correction term $m \mathbf{v}_{\mathbf{k}} \cdot \mathbf{v}_{\mathbf{k}}$ accounts for the shift in the reciprocal space vectors with deformation, which grows large near the zone boundary.

When more than one bands/pockets are present, interband/intervalley scattering must be accounted for. The matrix elements for inter-scattering are not obtainable without the details of the phonon spectrum and the Hamiltonian, which we lack because we are not studying a real system. Nevertheless, it is reasonable to assume that inter-scattering is generally weaker than intra-scattering because the wavefunction overlap between distinct bands or pockets is generally weaker than that within a band, and inter-scattering is often heavily reliant on zone-boundary phonons, which are less populated than zone-center phonons. To account for the strength of interscattering as such, we introduce a parameter, $s_{\text {int }}$ that acts as the lumped effect of the above-mentioned considerations. This quantity modulates the inter-scattering strength relative to intraband scattering. We set $s_{\text {int }}=0.5$ to reflect the usually weaker inter-scattering, which allows for trivial extension of Eq. (10) by accounting for the added phase space due to the second band proportional to its DOS. The overall DPS lifetime, for band 1, now becomes

$$
\tau_{\mathrm{DPS}, 1 \mathbf{k}}=\frac{\rho v_{s}^{2}}{\pi k_{\mathrm{B}} T\left(\Delta+m_{1} \mathbf{v}_{1 \mathbf{k}} \cdot \mathbf{v}_{1 \mathbf{k}}\right)^{2}}\left[D_{1}\left(E_{1 \mathbf{k}}\right)+s_{\text {int }} D_{2}\left(E_{1 \mathbf{k}}\right)\right]^{-1},
$$

where the subscripts 1 and 2 indicate each of the two bands. The addition of inter-scattering phase space is determined by the presence of second band states at given $E_{1}$. That is, if $D_{2}\left(E_{1 k}\right)=0$, then there is no inter-scattering.

In spite of its simplicity, the above formulations work well also for zoneboundary phonon scattering. For instance, $\mathrm{Fe}_{2} \mathrm{TiSi}$ conduction bands are very flat-and-dispersive, allowing significant zone-boundary phonon scattering as well as intervalley/interband scattering. Accurately calculated scattering rates of $\mathrm{Fe}_{2} \mathrm{TiSi}$ using DFT band structures and electron-phonon matrix elements ${ }^{71,72}$ behave essentially as $\tau^{-1}(E) \propto D(E)$ (see Supplementary Fig. $4 a)^{52}$, which is also the behavior predicted by Eqs. (10) and (11). The same conclusion holds for the flat-and-dispersive valence bands of $\mathrm{Li}_{2} \mathrm{TlBi}^{56}$. Therefore, the phenomenological treatment by Eqs. (10) and (11) capture the essence of intra- and inter-scattering even in cases with extreme anisotropy.

\section{Polar-optical scattering (POS)}

We modify the established formula for $\mathrm{POS}^{63,64}$ as to reasonably accounts for band anisotropy and inverted parabolicity

$\tau_{\mathrm{POS}, \mathbf{k}}=\frac{\left|\mathbf{v}_{\mathbf{k}}\right|}{2 \omega_{o}}\left(\frac{1}{\epsilon_{\infty}}-\frac{1}{\epsilon}\right)^{-1}\left[\left(b\left(\omega_{o}\right)+1\right) \cdot \sinh ^{-1}\left(\frac{D\left(E_{\mathbf{k}}-\omega_{o}\right)}{D\left(\omega_{o}\right)}\right)+b\left(\omega_{o}\right) \cdot \sinh ^{-1}\left(\frac{D\left(E_{\mathbf{k}}\right)}{D\left(\omega_{o}\right)}\right)\right]^{-1}$,

Modifications come from exchanging $\sqrt{E}$ with our tetrahedronintegrated $D(E)$ and using the $\mathbf{k}$-dependent prior form (instead of the less general E-dependent form-see Supplementary Discussion). Use of the group velocity norm $\left(\left|\mathbf{v}_{\mathbf{k}}\right|\right)$ obviates the dependence on $m$ and $E$ through the relation $\left|\mathbf{v}_{\mathbf{k}}\right|=\sqrt{\frac{2 E}{m}}$ for an isotropic parabolic band. The optical phonon frequency is represented by $\omega_{o}$, and $b\left(\omega_{o}\right)$ is the Bose-Einstein population. The left term in the square brackets accounts for phonon emission whereas the right term accounts for phonon absorption. For our band structures, Eq. (12) is exact in the upward-parabolic portions up to the inflection point, and past it, approximates the true lifetimes. When more than one band pocket exists at one k-point, nothing prohibits interband POS from occuring. To account for this, we take the same approach as we do with DPS and use $D(E)=D_{1}(E)+s_{\text {int }} D_{2}(E)$ to enlarge the phase space.

\section{lonized-impurity scattering (IIS)}

We use a modified version of the Brooks-Herring formula ${ }^{66,67}$ that reasonably account for band anisotropy and inverted parabolicity

$\tau_{\| I S, \mathbf{k}}=\frac{\epsilon^{2}|\mathbf{k}|^{4}}{2 \pi^{3} N_{i} Z^{2}} D^{-1}\left(E_{\mathbf{k}}\right)\left(\log \left(1+\gamma_{\mathbf{k}}\right)-\frac{\gamma_{\mathbf{k}}}{1+\gamma_{\mathbf{k}}}\right)^{-1}$,

where the screening term is

$\gamma_{\mathbf{k}}=\frac{4|\mathbf{k}|^{2} \epsilon k_{\mathrm{B}} T}{n}\left(\frac{F_{\frac{1}{2}}\left(E_{\mathrm{F}}\right)}{F_{-\frac{1}{2}}\left(E_{\mathrm{F}}\right)}\right)$.

Modification comes from using the $\mathbf{k}$-dependent prior form of the Brooks-Herring formula (instead of its typical energy-dependent formsee Supplementary Methods) and replacing the terms in it that represent the parabolic DOS with our tetrahedron-integrated $D(E)$. For our band structures, this corrected formula is again exact for the upward-parabolic portions from $\Gamma$ to the inflection point, and past it, closely approximates the true lifetimes. When more than one band pocket exists at the same k-point, interband IIS can take place. To account for this, we again use $D(E)=D_{1}\left(E_{1 \mathbf{k}}\right)+s_{\text {int }} D_{2}\left(E_{1 \mathbf{k}}\right)$ to modify the phase space. We assume that one impurity donates or accepts one charge, meaning the effective impurity charge of $Z=1$. This choice forces that the carrier concentration $(n)$ effectively equals the impurity concentration $\left(n=N_{i}\right)$ at appreciable doping levels.

\section{Materials parameters}

To calculate specific values of scattering, material-dependent quantities that control the relative strength of the three scattering mechanisms must be chosen. These include the deformation potential, the dielectric constants, and the optical phonon frequency among others. We select plausible values for these quantities that occur prevalently in real materials, as listed in Table 1. In emphasis, the choice of these values renders the relative strength of each scattering channel arbitrary. What is not arbitrary is the characteristic thermoelectric behavior of bands under a given scattering regime.

Table 1. Arbitrary quantities used in the scattering and transport models.

\begin{tabular}{lll}
\hline Arbitrary quantity & Symbol & Value \\
\hline Temperature & $T$ & $500 \mathrm{~K}$ \\
Density & $\rho$ & $5000 \mathrm{~kg} / \mathrm{m}^{3}$ \\
Sound velocity & $v_{s}$ & $4000 \mathrm{~m} / \mathrm{s}$ \\
Deformation potential & $\Delta$ & $0.4 \mathrm{Ha}=10.8 \mathrm{eV}$ \\
Interband scattering strength & $s_{\text {int }}$ & 0.5 \\
Static dielectric constant & $\epsilon$ & 30 \\
High-freq. dielectric constant & $\epsilon_{\infty}$ & 25 \\
Optical phonon frequency & $\omega_{o}$ & $k_{\mathrm{B}} T / 2$ \\
Effective charge of an impurity & $Z$ & 1 \\
Impurity concentration & $N_{i}$ & $n / Z$ \\
Lattice thermal conductivity & $K_{\text {lat }}$ & $0.5 \mathrm{~W} \mathrm{~m}^{-1} \mathrm{~K}^{-1}$ \\
\hline
\end{tabular}




\section{DATA AVAILABILITY}

The data can be either reproduced or generated using any user-desired parameters using publicly available Mathematica notebooks (see below).

\section{CODE AVAILABILITY}

The Mathematica notebooks are publicly available on the following link: https:// github.com/jsyony37/bandmodel.

Received: 17 October 2020; Accepted: 12 February 2021; Published online: 25 March 2021

\section{REFERENCES}

1. Snyder, J. \& Toberer, E. Complex thermoelectric materials. Nat. Mater. 7, 2 (2008).

2. Sootsman, J. R., Chung, D. \& Kanatzidis, M. New and old concepts in thermoelectric materials. Angew. Chem. 48, 46 (2009).

3. Zeier, W. G. et al. Thinking like a chemist: intuition in thermoelectric materials. Angew. Chem. 55, 24 (2016).

4. Zebarjadi, M., Esfarjani, K., Dresselhaus, M. S., Ren, Z. F. \& Chen, G. Perspectives on thermoelectrics: from fundamental to device applications. Energy Environ. Sci. 5, 5147 (2012).

5. Zhang, X. \& Zhao, L.-D. Thermoelectric materials: energy conversion between heat and electricity. J. Materiomics. 1, 2 (2015).

6. Zhu, T. et al. Compromise and synergy in high-efficiency thermoelectric materials. Adv. Mater. 29, 14 (2017).

7. Mao, J. et al. Advances in thermoelectrics. Adv. Phys. 67, 2 (2018),

8. He, J. \& Tritt, T. M. Advances in thermoelectric materials research: looking back and moving forward. Science 357, 6358 (2017).

9. Yang, J. et al. On the tuning of electrical and thermal transport in thermoelectrics: an integrated theory-experiment perspective. Npj Comput. Mater. 2, 15015 (2016).

10. Gorai, P., Stevanović, V. \& Toberer, E. S. Computationally guided discovery of thermoelectric materials. Nat. Rev. Mater. 2, 17053 (2017).

11. Jain, A., Shin, Y. \& Persson, K. A. Computational predictions of energy materials using density functional theory. Nat. Rev. Mater. 1, 15004 (2016).

12. Wang, H., Pei, Y., LaLonde, A. D., Snyder, G. J. Material Design Considerations Based on Thermoelectric Quality Factor, 3-32 (Springer, 2013).

13. Xin, J. et al. Valleytronics in thermoelectric materials. Quantum Mater. 3, 9 (2018).

14. Norouzzadeh, P. \& Vashaee, D. Classication of valleytronics in thermoelectricity. Sci. Rep. 6, 22724 (2016).

15. Witkoske, E., Wang, X., Lundstrom, M., Askarpour, V. \& Maassen, J. Thermoelectric band engineering: the role of carrier scattering. J. Appl. Phys. 122, 17 (2017).

16. Kumarasinghe, C. \& Neophytou, N. Band alignment and scattering considerations for enhancing the thermoelectric power factor of complex materials: the case of Co-based half-Heusler alloys. Phys. Rev. B 99, 19 (2019).

17. Maassen, C. R. J. Analysis of simple scattering models on the thermoelectric performance of analytical electron dispersions. J. Appl. Phys. 127, 6 (2020).

18. Zhou, J., Yang, R., Chen, G. \& Dresselhaus, M. S. Optimal bandwidth for high efficiency thermoelectrics. Phys. Rev. Lett. 108, 226601 (2011).

19. Jeong, C., Kim, R. \& Lundstrom, M. S. On the best bandstructure for thermoelectric performance: a Landauer perspective. J. Appl. Phys. 111, 113707 (2012).

20. Onsager, L. Reciprocal relations in irreversible processes I. Phys. Rev. 37, 405-426 (1931).

21. Onsager, L. Reciprocal relations in irreversible processes II. Phys. Rev. 38, 2265-2279 (1931)

22. Callen, $\mathrm{H}$. The application of Onsager's reciprocal relations to thermoelectric, thermomagnetic, and galvanomagnetic effects. Phys. Rev. 73, 11 (1948).

23. Jones, W., March, N. H. Theoretical Solid State Physics (Dover Books, 1985).

24. Ziman, J. M. Electrons and Phonons: the Theory of Transport Phenomena in Solids (Oxford University Press, 1960).

25. Scheidemantel, T. J., Ambrosch-Draxl, C., Thonhauser, T., Badding, J. V. \& Sofo, J. O. Transport coefficients from first-principles calculations. Phys. Rev. B. 68, 125210 (2003).

26. Madsen, G. K. \& Singh, D. J. BoltzTraP. A code for calculating band-structure dependent quantities. Comput. Phys. Commun. 175, 67-71 (2006).

27. Pei, Y., LaLonde, A. D., Wang, H. \& Snyder, G. J. Low effective mass leading to high thermoelectric performance. Energy Environ. Sci. 5, 7 (2012).

28. Yan, J. et al. Material descriptors for predicting thermoelectric performance. Energy Environ. Sci. 8, 3 (2015).

29. Gibbs, Z. M. et al. Effective mass and Fermi surface complexity factor from ab initio band structure calculations. Npj Comput. Mater. 3, 8 (2017).
30. $\mathrm{Fu}, \mathrm{C}$. et al. High band degeneracy contributes to high thermoelectric performance in p-type half-Heusler compounds. Adv. Energy Mater. 4, 1400600 (2014).

31. Zhang, J. et al. Designing high-performance layered thermoelectric materials through orbital engineering. Nat. Commun. 7, 10892 (2016).

32. Zhang, J. et al. High-performance pseudocubic thermoelectric materials from non-cubic chalcopyrite compounds. Adv. Mater. 26, 23 (2014).

33. Pei, Y., Wang, H. \& Snyder, G. J. Band engineering of thermoelectric materials. Adv. Mater. 24, 46 (2012).

34. Pei, Y., Xiaoya, S., Aaron, L., H. Wang, L. C. \& Snyder, G. Convergence of electronic bands for high performance bulk thermoelectrics. Nature 473, 7345 (2011).

35. Xia, Y., Park, J., Zhou, F. \& Ozolinš̌, V. High thermoelectric power factor in intermetallic CoSi arising from energy filtering of electrons by phonon scattering. Phys. Rev. Appl. 11, 2 (2019).

36. Pshenay-Severin, D. A., Ivanov, Y. V. \& Burkov, A. T. The effect of energydependent electron scattering on thermoelectric transport in novel topological semimetal CoSi. J. Phys.: Condens. Matter 30, 47 (2018).

37. Xia, Y., Park, J., Ozolinšs, V. \& Wolverton, C. Leveraging electron-phonon interaction to enhance thermoelectric power factor in graphene-like semimetals. Phys. Rev. B (R.) 100, 201401 (2019).

38. Lianga, J., Fana, D., Jianga, P., Liua, H. \& Zhao, W. First-principles study of the thermoelectric properties of intermetallic compound $\mathrm{YbAl}_{3}$. Intermetallics 87, 27-30 (2017)

39. Markov, M., Rezaei, S. E., Sadeghi, S. N., Esfarjani, K. \& Zebarjadi, M. Thermoelectric properties of semimetals. Phys. Rev. Mater. 3, 9 (2019)

40. Markov, M., Rezaei, S. E., Sadeghi, S. N., Esfarjani, K. \& Zebarjadi, M. Ultra-high thermoelectric power factors in narrow gap materials with asymmetric bands. J. Phys. Chem. C. 124, 33 (2020).

41. Mahan, G. D. \& Sofo, J. The best thermoelectric. Proc. Natl Acad. Sci. 93, 7436-7439 (1996)

42. Wang, H., Pei, Y., LaLonde, A. D. \& Snyder, G. J. Weak electron-phonon coupling contributing to high thermoelectric performance in $n$-type PbSe. Proc. Nat. Acad. Sci. 109, 25 (2012).

43. Zhang, X. et al. Electronic quality factor for thermoelectrics. Sci. Adv. 6, 46 (2020).

44. Torquato, S. Random Heterogeneous Materials (Springer, 2002).

45. $\mathrm{He}$, J. et al. $\mathrm{Bi}_{2} \mathrm{PdO}_{4}$ : a promising thermoelectric oxide with high power factor and low lattice thermal conductivity. Chem. Mater. 29, 6 (2016).

46. Isaacs, E. \& Wolverton, C. Remarkable thermoelectric performance in $\mathrm{BaPdS}_{2}$ via pudding-mold band structure, band convergence, and ultralow lattice thermal conductivity. Phys. Rev. Mater. 3, 1 (2019).

47. Kayser, P., Serrano-Sanchez, F., Dura, O. J., Fauth, F. \& Alonso, J. A. Experimental corroboration of the thermoelectric performance of $\mathrm{Bi}_{2} \mathrm{PdO}_{4}$ oxide and $\mathrm{Pb}$-doped derivatives. J. Mater. Chem. C. 8, 16 (2020).

48. Bilc, D. I., Hautier, G., Waroquiers, D., Rignanese, G.-M. \& Ghosez, P. Lowdimensional transport and large thermoelectric power factors in bulk semiconductors by band engineering of highly directional electronic states. Phys. Rev. Lett. 114, 13 (2015).

49. Dylla, M. T., Kang, S. D. \& Snyder, G. J. Effect of two-dimensional crystal orbitals on fermi surfaces and electron transport in three-dimensional perovskite oxides. Angew. Chem. 131, 17 (2019).

50. Kohn, W. \& Sham, L. J. Self-consistent equations including exchange and correlation effects. Phys. Rev. 140, 4A (1965).

51. Perdew, J. P., Burke, K. \& Ernzerhof, M. Generalized gradient approximation made simple. Phys. Rev. Lett. 77, 18 (1996).

52. Park, J., Xia, Y. \& Ozolinšs, V. High thermoelectric power factor and efficiency from a highly dispersive band in $\mathrm{Ba}_{2} \mathrm{BiAu}$. Phys. Rev. Appl. 11, 1 (2019).

53. Okuda, T., Nakanishi, K., Miyasaka, S. \& Tokura, Y. Large thermoelectric response of metallic perovskites: $\mathrm{Sr}_{1-x} \mathrm{La}_{x} \mathrm{TiO}_{3}(0 \leq x \leq 0.1)$. Phys. Rev. B 63, 11 (2001).

54. Okuda, T., Nakanishi, K., Miyasaka, S. \& Tokura, Y. High-temperature carrier transport and thermoelectric properties of heavily La- or $\mathrm{Nb}$-doped $\mathrm{SrTiO}_{3}$ single crystals. J. Appl. Phys. 97, 3 (2005).

55. Park, J., Xia, Y., Ganose, A., Jain, A. \& Ozolinš̌, V. High thermoelectric performance and defect energetics of multipocketed full Heusler compounds. Phys. Rev. Appl. 14, 2 (2020).

56. He, J., Xia, Y., Naghavi, S. S., Ozolinš̌, V. \& Wolverton, C. Designing chemical analogs to $\mathrm{PbTe}$ with intrinsic high band degeneracy and low lattice thermal conductivity. Nat. Commun. 10, 719 (2019).

57. Blöchl, P. E., Jepsen, O. \& Anderson, O. Improved tetrahedron method for Brillouin-zone integrations. Phys. Rev. B 49, 23 (1994).

58. Lu, X. et al. High performance thermoelectricity in earth-abundant compounds based on natural mineral tetrahedrites. Adv. Energy Mater. 3, 3 (2013).

59. Nielsen, M. D., Ozolinš̌, V. \& Heremans, J. Lone pair electrons minimize lattice thermal conductivity. Energy Environ. Sci. 6, 570-578 (2013).

60. Zhao, L. et al. Ultralow thermal conductivity and high thermoelectric figure of merit in SnSe crystals. Nature 508, 373-377 (2014). 
61. Nolas, G. S., Cohn, J. L., Slack, G. A. \& Scuhjman, S. B. Semiconducting Ge clathrates: promising candidates for thermoelectric applications. Appl. Phys. Lett. 73, 2 (1998).

62. Mukhopadhyay, S. et al. Two-channel model for ultralow thermal conductivity of crystalline $\mathrm{TI}_{3} \mathrm{VSe}_{4}$. Science 360, 6396 (2018).

63. Nolas, G. S., Sharp, J., Goldsmid, H. J. Thermoelectrics (Springer, 2001).

64. Lundstrom, M. Fundamentals of Carrier Transport (Cambridge University Press, 2000).

65. Fröhlich, H. Electrons in lattice fields. Adv. Phys. 3, 325-361 (1954).

66. Brooks, $H$. Theory of the electrical properties of germanium and silicon. Adv. Elec. Elec. Phys. 7, 85-182 (1955).

67. Chattopadhyay, D. \& Queisser, H. J. Electron scattering by ionized impurities in semiconductors. Rev. Mod. Phys. 53, 4 (1981).

68. Matthiessen, A. Ueber die elektrische Leitungsfähigkeit der Legirungen. Ann Phys. 186, 6 (1860).

69. Bardeen, J. \& Shockley, W. Deformation potentials and mobilities in non-polar crystals. Phys. Rev. 80, 1 (1950).

70. Khan, F. S. \& Allen, P. B. Deformation potentials and electron-phonon scattering: two new theorems. Phys. Rev. B 29, 6 (1984).

71. Giustino, F., Cohen, M. L. \& Louie, S. G. Electron-phonon interaction using Wannier functions. Phys. Rev. B 76, 16 (2007).

72. Ponce, S., Margine, E. R., Verdi, C. \& Giustino, F. EPW: electron-phonon coupling, transport and superconducting properties using maximally localized Wannier functions. Comput. Phys. Commun. 55, 116-133 (2016).

\section{ACKNOWLEDGEMENTS}

This work was led by funding from U.S. Department of Energy, Office of Basic Energy Sciences, Early Career Research Program, which supported J.P. and A.J. Lawrence Berkeley National Laboratory is funded by the Department of Energy under award DE-AC02-05CH1 1231. V.O. acknowledges financial support from the National Science Foundation Grant DMR-1611507. This work used resources of the National Energy Research Scientific Computing Center, a Department of Energy Office of Science User Facility supported by the Office of Science of the U.S. Department of Energy under Contract No. DE-AC02-05CH11231. J.P. thanks Younghak Kwon of UCLA Mathematics for helpful discussions.

\section{AUTHOR CONTRIBUTIONS}

J.P. developed the methods, implemented the codes, and analyzed results. A.J. supervised the study. All authors helped design the study, interpret results, as well as draft and revise the paper.

\section{COMPETING INTERESTS}

The authors declare no competing interests.

\section{ADDITIONAL INFORMATION}

Supplementary information The online version contains supplementary material available at https://doi.org/10.1038/s41524-021-00512-w.

Correspondence and requests for materials should be addressed to J.P. or A.J.

Reprints and permission information is available at http://www.nature.com/ reprints

Publisher's note Springer Nature remains neutral with regard to jurisdictional claims in published maps and institutional affiliations.

(i) Open Access This article is licensed under a Creative Commons Attribution 4.0 International License, which permits use, sharing, adaptation, distribution and reproduction in any medium or format, as long as you give appropriate credit to the original author(s) and the source, provide a link to the Creative Commons license, and indicate if changes were made. The images or other third party material in this article are included in the article's Creative Commons license, unless indicated otherwise in a credit line to the material. If material is not included in the article's Creative Commons license and your intended use is not permitted by statutory regulation or exceeds the permitted use, you will need to obtain permission directly from the copyright holder. To view a copy of this license, visit http://creativecommons. org/licenses/by/4.0/.

(c) The Author(s) 2021 